Research Article

\section{Coronavirus COVID-19 surface properties: Electrical charges status}

\section{Luisetto $\mathbf{M}^{1 *}$, Tarro $\mathbf{G}^{2}$, Khaled Edbey ${ }^{3}$, Farhan Ahmad Khan ${ }^{4}$, Ahnaf IIman ${ }^{5}$, Yesvi AR ${ }^{6}$, Nili BA ${ }^{7}$, Fiazza ${ }^{8}$, Mashori GR $^{9}$ and Latyshev OY10}

${ }^{1}$ IMA, Marijnskaya Academy, Vice President, Environmental Toxicology, Natural Science Branch, Applied Pharmacologist, I29212, Italy

2President, T \& L de Beaumont Bonelli Foundation for Cancer Research, Naples, Italy

${ }^{3}$ Professor, Department of Chemistry, University of Benghazi, Libya

${ }^{4}$ Professor, Department of Pharmacology, J.N. Medical College, AMU, Aligarh, India

${ }^{5}$ Student, Dhaka Residential Model College, Dhaka, Bangladesh

${ }^{6}$ Founder and President, Yugen Research Organization, Undergraduate Student, Western Michigan University, MI, USA

${ }^{7}$ Innovative Pharmaceutical Product Development Specialist, USA

${ }^{8}$ Medical Pharmacologist, Independent Researcher, Italy

${ }^{9}$ Profesor, Department of Medical \& Health Sciences for Woman, Peoples University of Medical and Health Sciences for Women, Pakistan

${ }^{10}$ President, IMA Academy, Italy

\section{Abstract}

Aim of this work is to analyze the coronavirus viral surface properties related the pattern of electrical features.

This chemical physical property is relevant and crucial to set profile of diffusion, severity of disease, efficacy of therapeutic strategy and in order to search new way to fight COVID-19 and the NEW VARIANT.

The phenomena of immune evasion and the different pattern of efficacy towards variants of some vaccine or some antibodies combination produce the need to verify if considering the electrical feature of viral surface can be a right tool or not.

As result of this research it is possible to submit to the scientist that the viral surface properties and electrical feature can be an element to be considered in various preventive or treatment measure.

The specificity of action of some vaccine or antibodies seem to tell us that also the aspecific methods are useful.

A specific chemico physical factors can influence the electrical charges viral surface behavior.

Hpertonic saline solution, humidity, electrical charge barrier in mask are simply example of the effect.

That can be obtained action on viral surface chemico -physical properties.

\section{More Information}

*Address for Correspondence: Luisetto Mauro, IMA, Marijnskaya Academy, Vice President Environmental Toxicology, Natural Science Branch, Applied Pharmacologist, 129212, Italy, Tel: +393402479620; Email: maurolu65@gmail.com

Submitted: March 24, 2021

Approved: April 12, 2021

Published: April 13, 2021

How to cite this article: Luisetto M, Tarro G, Edbey K, Khan FA, IIman A, et al. Coronavirus COVID-19 surface properties: Electrical charges status. Int J Clin Microbiol Biochem Technol. 2021; 4: 016-027.

DOI: 10.29328/journal.ijcmbt.1001021

Copyright: @ 2021 Luisetto M, et al. This is an open access article distributed under the Creative Commons Attribution License, which permits unrestricted use, distribution, and reproduction in any medium, provided the original work is properly cited.

Keywords: Coronavirus; COVID-19; Envelope; Electrical charge; Severity of disease; Spread Surface chemical physical properties; New strategy; Specific and aspecific measure

Check for updates

OPEN ACCESS

\section{Introduction}

The emerging of new COVID-19 VARIANT is a great health public problem:

According an article Oxford COVID-19 vaccine trial results

7 February 2021 - Wits University.
The Wits Vaccines and Infectious Diseases Analytics (VIDA) Research Unit, which runs the Oxford COVID-19 vaccine trial in South Africa, has announced results:

"In an analysis, submitted as a pre-print prior to peerreview publication, a 2-dose regimen of the;

ChAd0x1 nCoV-19 vaccine provides minimal protection 
against mild-moderate COVID-19 infection from the B.1.351 coronavirus variant first identified in South Africa SA in midNovember 2020."

"Researchers from South Africa and the UK found that viral- neutralization by sera induced by the ChAdOx1 nCoV-19 coronavirus vaccine against the B.1.351 coronavirus variant were substantially reduced when compared with the original strain of the coronavirus."

And according an article of Hannah Balfour (European Pharmaceutical Review) 28 January 2021.

News: "Study finds some antibody- therapies are not effective against new COVID-19 variants".

According the article of 2021: Chemico-physicals properties of coronavirus affecting.

Airborne transmissibility: "Coronavirus was finded to have characteristic pattern of chemical -physical properties at $\mathrm{X}$-ray.

\section{Cristallograpy analysis}

So not only carrier size is relevant for airborne transmission of some respiratory virus but also virus size and electricalfeature, envelope composition and other. The virus envelope seem involved in avoiding strategies of virus to escape to the hosts immune -system".

Because coronavirus follow determinate pattern of transmission it is crucial to observe the chemical-physical properties of the virus as the electrical- feature, molecular composition of the envelope, viral- size and physical effect that can increase airborne characteristic in aerosols. The same the kind of chemical physical link of viruses with carrier is relevant for its diffusion.

All this properties must be taken in consideration in the same evaluation process.

Even if the virus transmission depend on determinate viral charge and other relevant factors a better understanding of this related phenomena is crucial. (It must be remembered also that the smaller -particles like aereosols penetrate better lower pulmonary -tract then the larger one).

Common properties among some respiratory- virus can help in clarifying airborne- transmission: If already clearly for some common virus for other it must be finally determinate. This conclusion present great health implication in public policy.

Piotr H Pawłowski: "The charged amino- acids in the spike protein of SARS-CoV-2 exhibit some specific distribution. In the RBD region of the $\mathrm{S} 1$ unit the positive + charge dominates in the protein interior and the negative - charge prevails on the surface exposed towards ACE2 -receptor. The charged amino- acids in the $\mathrm{S} 2$ region preceding heptad repeats of the spike protein of SARS-CoV-2, exhibit a central concentration, and the imbalance of a positive + charge. The idea that both distributions of charged amino acids may, in an electrostaticmanner, facilitate the coronavirus infection of the cell is presented.

An evaluation of the virus-receptor binding energy, by docking the peptide resembling the human receptor site to the virus- RBD, and the estimation of electromechanical deformation of the cell membrane by S2 during the prefusion process were included. Possible prevention of viral- infection is suggested."

\section{And}

"Although the total structural charge of SARS-Cov-2 is positive + , the structural proteins of SARS-Cov-2 are carrying varied total electric charge related to the amino- acid AA content. The envelope, membrane and nucleocapsid proteins are positive + , and the surface spike protein $S$ is negative. The viral spike -protein $S$ is an essential component in the process of SARS-Cov-2 entering the cell. It contains 99 positively, and 111 negatively, charged amino- acids. Protein S consists with the two subunits S1 and S2. The S1 unit (aa 14-685) contains the receptor binding -domain (aa 333-526), RBD, interplaying with the human angiotensin-converting enzyme 2, ACE2, during virus binding to the cell. Enzymatic- cleavage in the area aa 682-685 in fully reveals subunit S2. Some segments of the S2 unit (aa 686-1273) play an important role in the fusion of SARS-CoV-2 preceding the underlying infection of cells. Although the exact location of the segments, enabling initial lipid- membrane boarding can be disputed, the investigation in the conformational conserved area of so called fusion peptide (FP), and in its close proximity seems to be rational. Similar regions to SARS-CoV Extended Bipartite Fusion- Platform, consisting with 38 amino acids perturbing membrane order in the two neighboring -regions, FP1 and FP2, may be also indicated, but they follow the FP (aa 788-806) of SARS-CoV-2 towards C-term".

\section{Materials and methods}

Whit an observational method some relevant literature are analysed and after submitting of an experimental project hipotesys a global conclusion is produced.

All literature come from biomedical database (PubMed) OR other open science source.

In this work also some preprint is reported for the scope researched.

Figures from 1 to 12 are reported in order to better clear the concept in literature cited.

\section{Results}

"The (pKa)s of the protein on the surface: At the isoelectricpoint of a protein, there is an equivalent distribution of 
negative and positive- charges, leading to a neutral response to a potential difference. If the $\mathrm{pH}$ be lowered from the isoelectric- point, it is found that, a bias is created towards the centre of positive charge of the protein. The pKa's could be derived from the $\mathrm{pH}$ values of the titration- curve for the $\mathrm{S}$ (spike)-protein of the SARS-CoV-2 using the HendersonHasselbalch equation:

$$
\mathrm{pH}=\mathrm{pKa}+\log ([\text { conjugate base }] /[\text { weak acid }]) .
$$

To establish this concept of the influence of surrounding $\mathrm{pH}$ on the surface charge of a virus, similar experiments had already been carried out also over the other virus- strains" [1].

Coronavirus envelope- protein: current knowledge Dewald Schoeman \& Burtram C. Fielding. Virology Journal volume 16, Article number: 69.

"a substantial portion of the TMD consists of the 2 nonpolar, neutral amino acids, valine and leucine, lending a strong hydrophobicity to the E protein. The peptide exhibits an overall net- charge of zero, the middle region being uncharged and flanked on one side by the negatively charged amino (N)-terminus, and, on the other side, the carboxy (C)terminus of variable charge. The C-terminus also exhibits some hydrophobicity but less than the TMD due to the presence of a cluster of basic, positively- charged amino acids" [2].

Science News from research organizations.

\section{Research exposes new vulnerability for SARS-CoV-2.}

Electrostatic interactions enhance the spike protein's bond to host cells.

"Using nanometer-level simulations, researchers have discovered a positively + charged site (known as the polybasic cleavage- site) located 10 nanometers from the actual binding

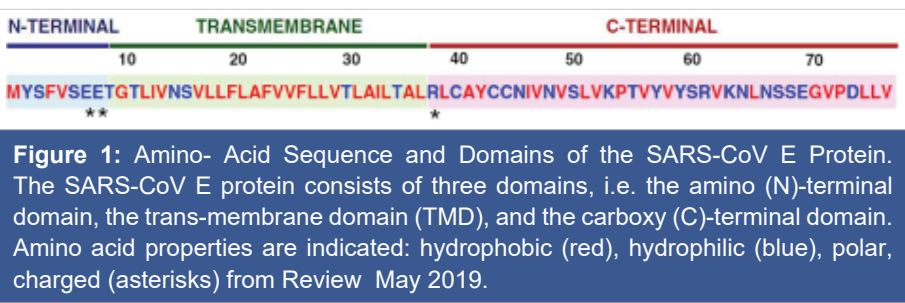

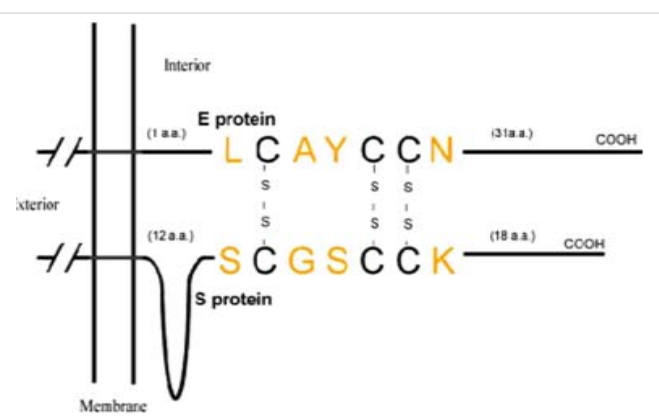

Figure 2: Predicted interaction between SARS-CoV E and $S$ proteins through disulphide bonds. site on the spike protein. The positively charged site allows strong bonding between the virus protein and the negativelycharged human-cell receptors" [3].

Edris Joonaki, et al: "By using the multilayer/multi-module approach using either small-diameter nano-fibers with small basis weight in the modules, or large-diameter nano-fibers with large basis weight in the modules, high-performance charged nanofiber filters have been developed that achieve over $90 \%$ capture of airborne COVID-19 simulated by the 100 $\mathrm{nm}$ sodium chloride- aerosols. If the coronavirus is negatively charged, the positively charged PVDF nanofiber filter will provide even higher capture efficiency as well by Coulomb attraction" [4].

The electrostatic- potential (Poisson-Boltzmann method) at the interface of SARS-CoV-2 spike receptor-binding domain (RBD) and human ACE2. Crystal structure 6M0J was analyzed. The meaning of color area: red-negative, and violetpositive, electric- potential. Yellow and blue lines represent ACE2, and RBD backbone. The examples of possible border regions which may interact via Coulomb forces are indicated by arrows. They represent positive + Lys and negative Glu at the RBD gate, producing electric dipole, tangential to the RBD-ACE2 interface plane. Computed with Swiss-Pdb Viewer v4.1.0. the idea In a case I, considering the possible inhibition of the virus-receptor binding, in the region of the RBD-ACE2
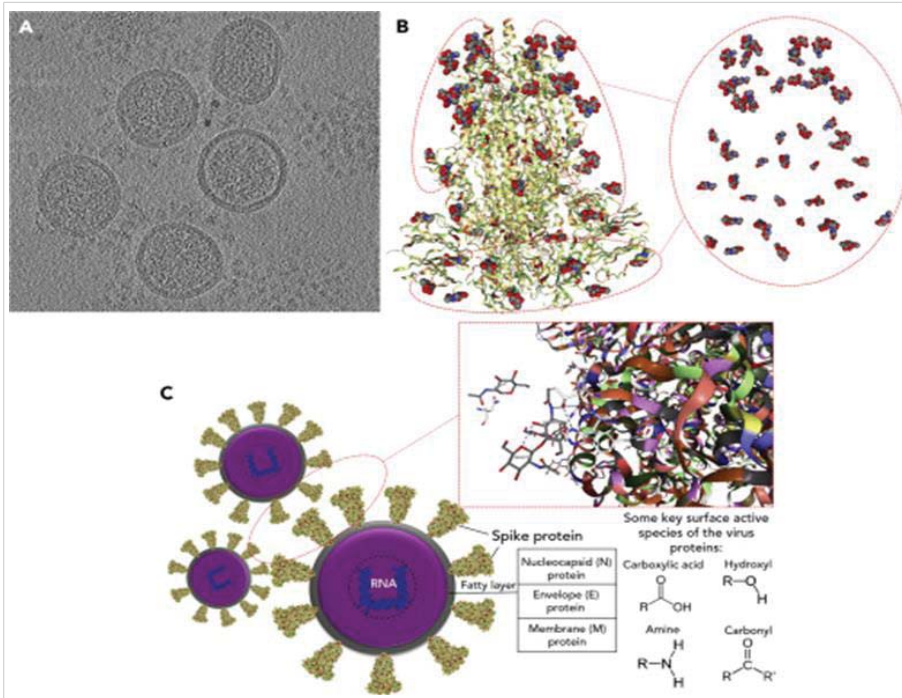

Figure 3: Structural- Chemistry and Identifying Surface-Active Species of the Virus(A) A central- slice through a cryo-EM tomogram of mouse hepatitis -virus for showing the existence of $S$ on the outer surface of virions. (B) Structure of the SARS-CoV-2 spike- glycoprotein with highlighting corresponding functional- groups (protein- database [PDB]: 6VXX). Grey, blue, and red spheres are carbon, nitrogen, and oxygen atoms, respectively. The molecular- structure is colored based on hydrophobicity; colored from red (hydrophilic) to green (hydrophobic). It utilizes the experimentally attained hydrophobicity scale that relied on whole-residue free energies of transfer DG (kcal/mol) from water to 1-Palmitoyl-2-oleoyl-phosphatidylcholine (POPC) interface. (C) Model representation of SARS-CoV-2 with respective proteins assembly. Key molecular- interactions among proteins on the surface of the virus -particle are shown as gray dash lines, "hydrophobic interactions," and blue dash lines, "hydrogen bonding (-OH----O)," (PDB: 6VYB). Some key surfaceactive moieties of SARS-CoV-2 are denoted as hydroxyl, amine, carbonyl, and carboxylic- acid functional groups FROM :Surface Chemistry Can Unlock Drivers of Surface Stability of SARS-CoV-2 in a Variety of Environmental Conditions. 


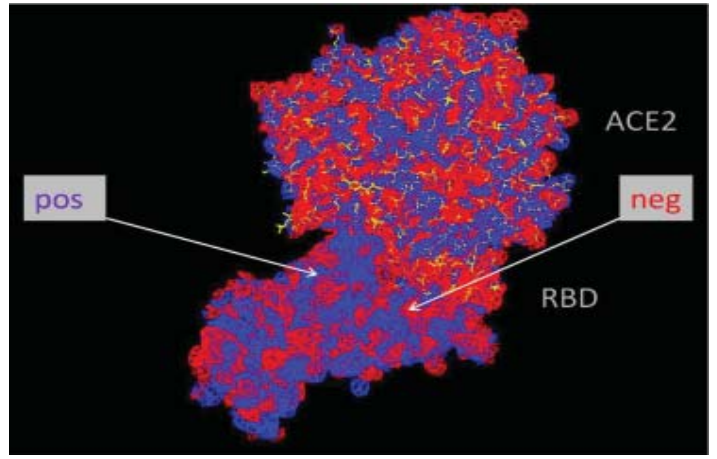

Figure 4: Form Piotr H. Pawłowski. (2021.) Charged amino acids may promote coronavirus SARS-CoV-2 fusion with the host cell[J]. AIMS Biophysics.

docking site, the cationic- ligands should be preferred, or the peptides with the specific charge distribution.

A precise binding of the RBD with the ligand also require the other types of physical- interactions, mainly hydrogen bonding, dominating the total binding energy. In a case II, considering the possible inhibition of prefusion, the negative charge of amino acid AA residues may be in pairs associated with extracellular $\mathrm{Ca}^{2+}$ cations in the vicinity of the cell membrane surface, and thus partially neutralized. Amino- acids with not compensated positive charge, located relatively closer to the cell-membrane, can be electrostatically attracted towards extracellular membrane leaflet, and finally mechanically compress the cell membrane, changing the phospholipids order.

The electric- field near the extracellular- surface driving positive + charges towards the cell surface is predicted by the total summary model, Goldman/Hodgkin/Katz Donnan/ Nernst-Ohki. Thus, inhibiting small anionic ligands for FP should be preferred.

And from Wallace Woon Fong Leung and Qiang qiang Sun:

Capture efficiency for single-layer filters with different fiber diameters.

"We examine the added effect of electrostatically charged fibers with different fiber diameters.

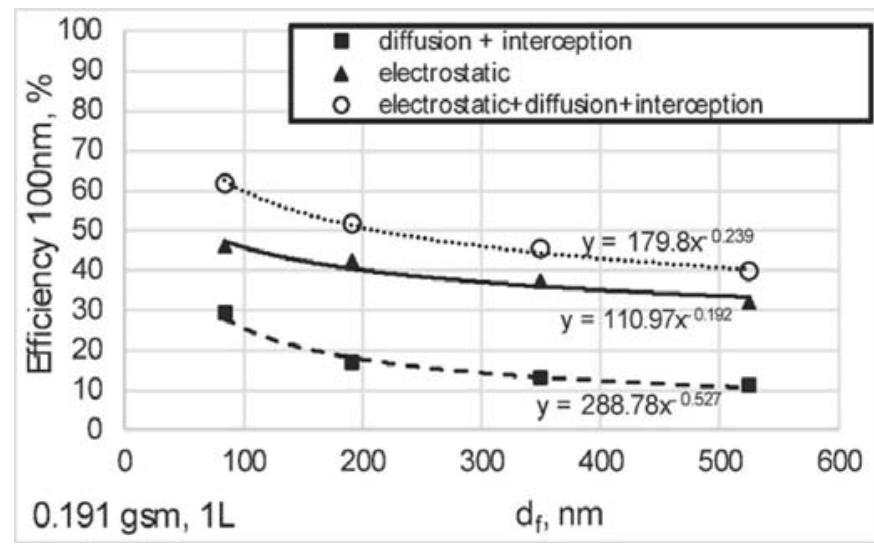

Figure 5
Breakdown of efficiency due to mechanical- diffusion plus interception and electrostatic effect for different nanofiberdiameters for a single layer with $0.191 \mathrm{gsm}$, the difference between these two curves with the same fiber -diameter represents only the electrostatic capture for a given aerosol size. Electrostatic- effect contributed much higher efficiency. This is in as much as $30 \%$ absolute and $100 \%$ on relative basis for a given fiber diameter" [4].

K Sholanov: "For further analysis of virus electricalproperties, an import role belong to the research results of scientists believing that genome of COVID-19 germ is recombinant (hybrid) virus composed of the SARS-CoV-2 virus and the second one that has ho equivalents. In this case, before entering human -body COVID-19 germ can be considered as impurity bio-polymer consisting of aliovalent molecules. This creates conditions for the emergence of electrical conductivity properties in the polymer as a consequence of the emergence of free electrostatic- charges. One method of creating conductive polymers is formation of a molecular- complex with chargetransfer between molecules forming impurity.

Depending on the structure of the components, different arrangement of hybrid virus- molecules is possible. It can be assumed that donors and acceptors form separate RNAchains. Donor- groups increase the cations number and acceptor- groups increase the anions number. Thus, impurity bio-polymer acquires an electrostatic- charge. The presence of virus electrostatic- charge is confirmed by some observations researchers from NIH, CDC, UCLA and Princeton- University found that the virus is stable during different periods in the aerosol and on the surfaces of different- materials.

Although, the experiments in question were performed by the researchers for other purposes, results of these experiments can be considered in terms of contact -electrification of the virus by various bodies. It is known that during the contact- electrification, double electric- layer is formed with different potential of surface- charges. Charge transfer between contacting- bodies ends when the intensity value caused by the field becomes equal to the field value conditioned by the difference between the activities of the output of contacting materials charged -particles. If the virus does not have an electric -charge, then it would be stable for a long time in all materials. Duration of charged particles exit from the hybrid -virus is determined by the difference in band structure of the contacting- body. The presence of a charge in the COVID19 virus germ assumes that there exists the next transfer mechanism: due to the emergence of the potential difference between human- body and the virus, the ionizedvirus is attracted to human -body surface and then enters human body.

High contagiousness of COVID-19 can be explained by the presence of the transfer mechanism of the virus that uses air ions. Air -ions are generated by air -ionization. At the same 
time, the negative air- ions are mostly created by oxygen, and the positive ones are mostly created by carbonic acid- gases. Air ions interact and link up viral ions with opposite signs, and form agglomerate of COVID-19 viruses in the air, which predominantly via respiratory- system get into the lungs. This explains the reasons of extreme contagiousness of the COVID-19 virus.

A natural question arises on how air is ionized. Special devices are used to ionize air, although in this case for the ionization on a global scale it is possible to use long-wave ultraviolet- radiation (315-400 $\mathrm{nm})$.For such UV- radiation formed, by an artificial -satellite, the ozone -screen of the Earth is not an obstacle. Thus, virus and air ions agglomerate formed by UV- radiation from the artificial satellite using, perhaps, laser equipment may cause large-scale coverage of COVID-19 population all over the planet.

Experience has shown that the disease is not always transmitted from person to person, the disease often appears in a person spending time- outdoors, which is due to inhalation of virus- agglomerate. At the same time, concentration of the agglomerate can be varied by varying the degree of airionization" [5].

From preprint Wei Li: "Among the four COVID-19 Coronavirus-related structures, three were determined using X-ray crystallography and one using electron- microscopy (PDB ID:6VSB)

After the four structures were accessed and downloaded directly from the PDB website, a comprehensive set of salt bridging analysis was carried out as described in previously.

No hydrogen bonding network analysis was carried out here because usually hydrogen atoms are missing from experimentally determined bio-molecular structures solved using X-ray crystallography. With the electrostatic analysis as described previously in, this article puts forward a comprehensive set of electrostatic- interaction features for the four COVID-19 coronavirus-related structures as of March 4,2020 , all included as a large set of separate tables in the supplementary file supplementary.pdf, including specifically:

1. all salt -bridges formed within all four COVID-19 coronavirus-related structures as of March 4, 2020.

2. all interfacial salt -bridges formed within all four COVID-19 coronavirus-related structures as of March 4, 2020.

3. all salt- bridges formed within all four COVID-19 coronavirus-related structures as of March 4, 2020, PDB ID-specifically for all four COVID-19 coronavirus-related structures.

4. all interfacial salt- bridges formed within all four COVID-19 coronavirus-related structures as of March 4, 2020, PDB ID-specifically for all four COVID-19 coronavirus-related structures.
No side chain or main chain hydrogen- bond was structurally identified for the four COVID-19 coronavirusrelated structures, as shown in supplementary file supplementary.pdf For the first time, this article reports a comprehensive set of electrostatic- features sucked out of the currently available COVID-19 coronavirus-related structures inside PDB in both PDF format and also LATEX format, i.e., a series of machine-readable -importable and -analyzable.

In supplementary file scan.zip, a simple python-based analysis tool and a LATEX-based editing tool were included to extract and summarize the electrostatic -features from experimentally determined structures, where a set of PDB files (representing experimentally determined protein structures) are to be plugged into a set of python- scripts included in cmd.py. Afterwards, cmd.py is to be executed with a simple command (python cmd.py) on a Linux machine terminal to produce the final supplementary file supplementary.pdf, summarizing the structurally observed electrostatic- features of the four COVID-19 coronavirus-related experimental structures as of March 4, 2020.

Along with the python-based analysis tool and a LATEXbased editing tool, the structurally observed electrostaticfeatures of the four COVID-19 coronavirus-related experimental structures constitute a preliminary starting point pointing towards a clear, coherent and comprehensive map of COVID-19's structure and function and also machinelearning and structure-based computationa-l design of neutralizing antibodies and/or small molecule(s) as potential therapeutic candidates against future outbreaks of the COVID-19 coronavirus -diseases" [6].

Jeffrey Baron, et al: "Bronchiolitis, an infection of the lower respiratory- tract, is the leading cause of infant and child hospitalization in the United States US. Therapeutic options for management of bronchiolitis are limited. Hypertonic saline inhalation -therapy has been studied in numerous clinical trials with mixed results. In 2014, the American Academy of Pediatrics (AAP) published updated guidelines on the diagnosis and management of bronchiolitis, which include new recommendations on the use of hypertonic -saline. We reviewed all published clinical trials mentioned in the 2014 AAP guidelines, as well as additional trials published since the guidelines, and critically evaluated each trial to determine efficacy, safety, and expectations of hypertonic- saline inhalation therapy. A total of 2682 infants were studied over the course of 22 clinical trials. Nine trials were carried out in the outpatient/clinic/emergency department and 13 in the inpatient setting. We agree with the AAP guidelines regarding the recommendation to use nebulized hypertonic -saline for infants hospitalized with bronchiolitis, with the expectation of reducing bronchiolitis scores and length of stay when it is expected to last more than 72 hours. We also believe there might be an advantage for hypertonic saline in reducing admission -rates from the emergency department, based on 
close examination of the results of recent trials. This review also highlights important gaps in the available literature that need to be addressed in order to define the role of inhaled hypertonic- saline therapy" [8].

Irina V Biktasheva: "Transient local over-dry environment might be a contributor and an explanation for the observed asynchronous local rises in Covid-19 mortality. We propose that a habitat's air- humidity negatively correlate with COVID-19 morbidity and mortality, and support this hypothesis on the example of publicly available data from German federal -states" [9].

"The virus SARS-CoV-2 is termed due to of its genome sequence similarity to SARS Coronavirus (SARS-CoV). The CoVs belong to the same genus Beta -coronavirus, sharing similar morphology in the form of enveloped, positive singlestranded RNA viruses. These viruses can be deactivated by certain lipid- solvents such as ethanol, ether (75\%), chlorine-containing disinfectants, and chloroform, except chlorhexidine. Ethyl alcohol, at concentrations of $60 \%-80 \%$, is a potent viricidal agent inactivating all the lipophilic viruses (influenza, herpes, vaccinia virus) and many hydrophilicviruses (adenovirus, enterovirus, rhinovirus, and rotaviruses but not hepatitis A virus (HAV) or poliovirus)" [10].

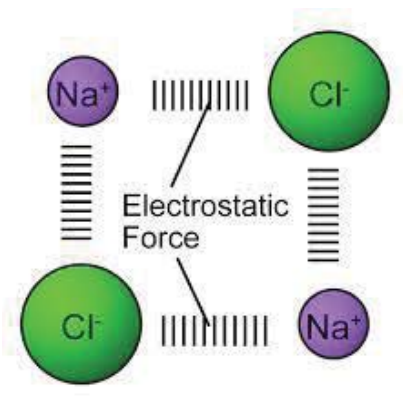

\section{Figure 6}

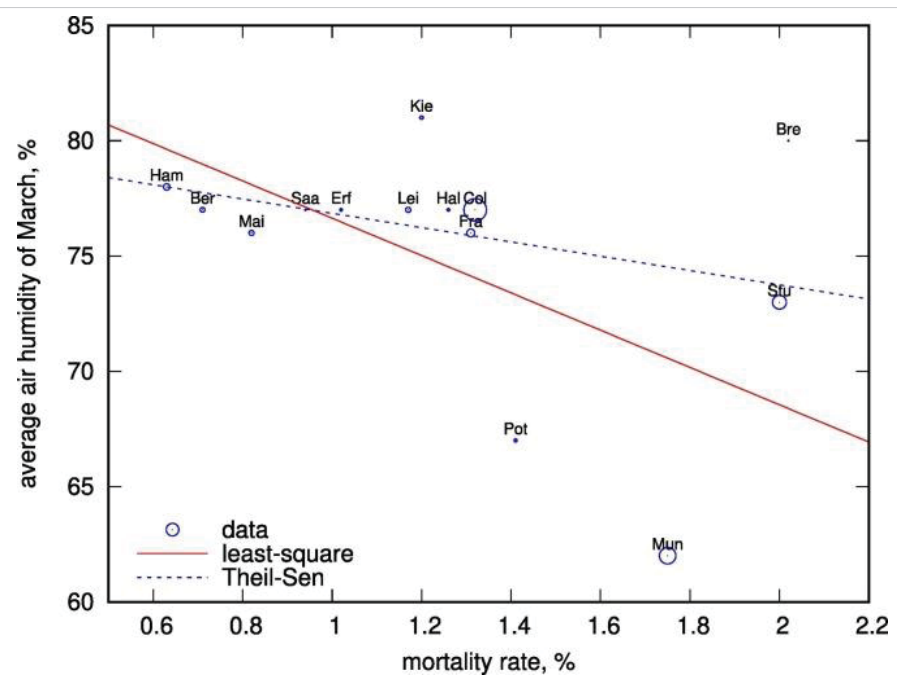

Figure 7: Air- humidity in March vs COVID-19 mortality in German federal- states (circles). The size of the circles is proportional to the population of the federal- state; the labels are abbreviations for the largest cities of the federal lands. Red solid line shows the linear regression. Theil-Sen regression is shown with the blue dashed -line.

\section{Experimental project hypotesys}

In order to verify the effect of modify the ionic forces in interface of an animal virus model it is interesting to use 2 different experimental condition:

Model A normal condition: respiratory virus and its bindigns on pulmonary epithelial cells

Mode B alternated condition: respiratory virus and its bindig under effect of different substantia that can modify the distribution of electrical charges in the interface virus epithelial cells.

The substantie used must to be not so toxic for the epithelial cell.

Time of observation: basal time and after 2 hours.

Endpoint: respiratory disease: yes or no (clinical and instrumental).

Kind of subministration: by aeresosl .

Kind of molecules studied: various mechanism of action but able to modify electrical charge pattern in virus surface.

Ethical consideration: under all international rules.

\section{Discussion}

Related the literature reported it is possible to say that:

The viral surface is relevant $t$ phenomena like transmissibility, airborne characteristics, bind with pulmonary epithelial cells, link whit PM particlate matter and also to the severity of the disease.

Phenomena like immune escape, variant emerging and variability in efficacy of vaccine (towards variants) and antibodies depends on the condition of viral surface.

Masks with electrical charge barriers show more efficacy.

Interfacies phenomena are relevant for the binding of the virus to the epithelial cells.

Aereosol therapy whit hypertonic saline solution show reduction in some pediatric respiratory viral disease (in number of days of pathology).

Air humidity condition if a factor that influence COVID -19 diffusion.

Electrical charges are involved in ligad between spike protein and ACE receptor.

Mahmoud Al Ahmad, et al: we identify and quantitate two similar viruses, human and feline- immunodeficiency viruses (HIV and FIV), suspended in a liquid medium without labeling, using a semiconductor- technique. The virus count 
C.

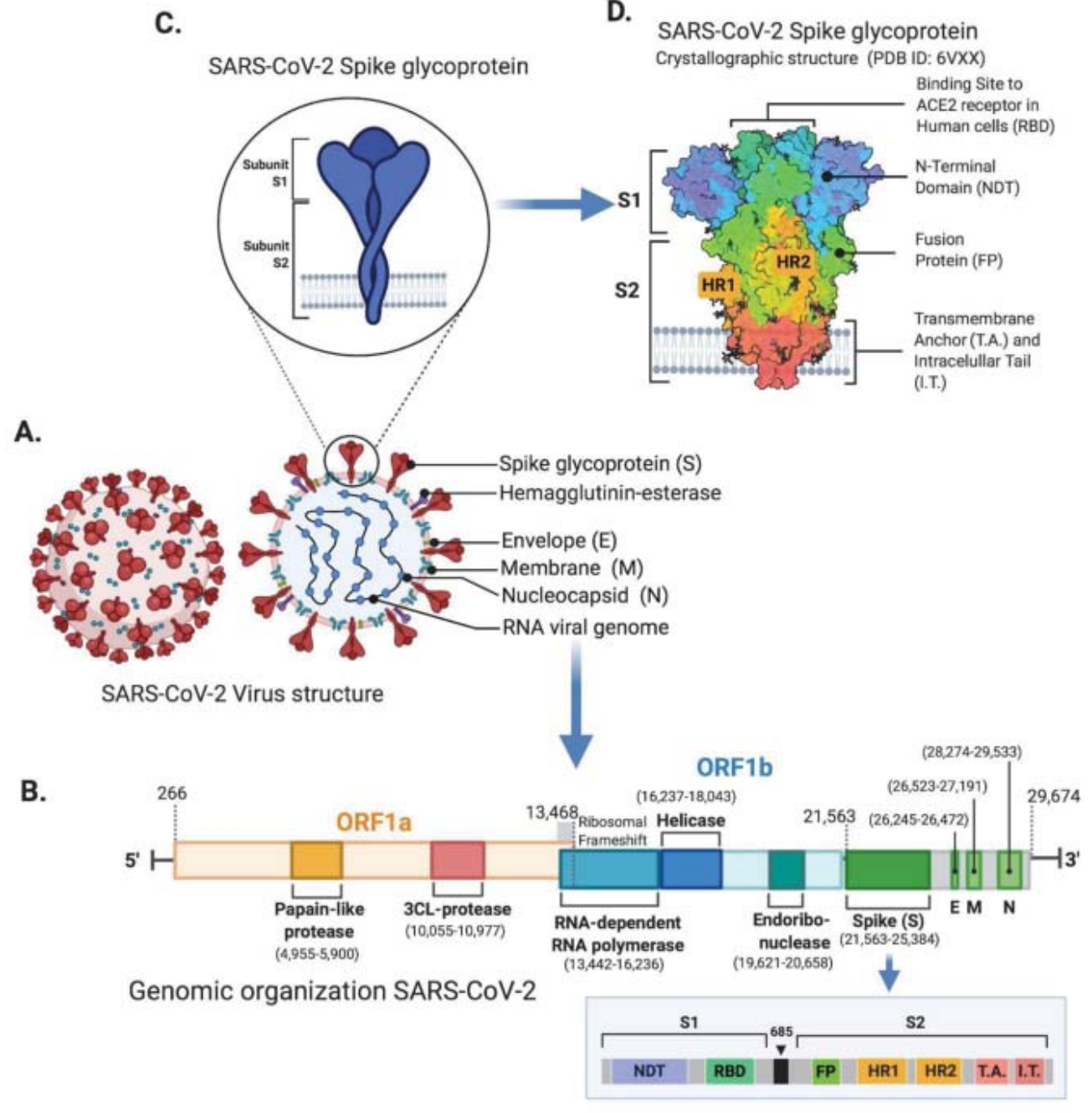

Figure 8: From Rameswari Chilamakuri, et al. Illustration of the rationale of the electrical measurement for virus titer measurement and classification. (a) Virions -distributions inside the coaxial resonator in the absence of electric -field. (b) Polarized virions when an electrostatic-c field is applied. A coaxial resonator has an inner- conductor (the +ve electrode, where positive charges accumulate, $+Q$ ) surrounded by a hollow space that is surrounded by a conducting shield (-ve electrode, where the negative charges accumulate, $-Q$ ). (c) Schematic of the polarized virus particles inside an alternating current electric- field.

was estimated by calculating the impurities inside a defined volume by observing the change in electrical -parameters. Empirically, the virus count was similar to the absolute value of the ratio of the change of the virus suspension dopant concentration relative to the mock dopant over the change in virus- suspension Debye volume relative to mock Debye volume. The virus type was identified by constructing a concentration-mobility relationship which is unique for each kind of virus, allowing for a fast (within minutes) and labelfree virus quantification and identification. For validation, the HIV and FIV virus preparations were further quantified by a bio-chemical technique and the results obtained by both approaches corroborated well. We further demonstrate that the electrical-technique could be applied to accurately measure and characterize silica- nanoparticles that resemble the virus particles in size. Based on these results, we anticipate our present approach to be a starting point towards establishing the foundation for label-free electrical-based identification and quantification of an unlimited number of viruses and other nano-sized particles. the methodology presented here is a basic attempt to demonstrate and explore the use of a new technique for virus- detection, classification and identification. The outlined technique should be applicable to any type of virus, provided it can be electrically- polarized, as well as other types of particles in suspended solutions" [12].

Robert I Mac Cuspie, et al: Capacitances of 5 types of viruses, adenovirus type 5 (AV 5) herpes simplex virus type 1 (HSV1), simian virus 40 (SV40), vaccinia (MVA), and cowpea mosaic- virus (CPMV) were compared by AC capacitance scanning probe microscopy. This technique, using a Pt-coated AFM tip as an electrode to probe capacitance of materials between the tip and a bottom- electrode, has been applied to study surface structures of semiconductors and polymers with nanometer spacial resolution, biological samples at the nanoscale have not been explored by this technique yet. Because most biological- cells are poor conductors, this 
approach to probe electric properties of cells by capacitance is logical. This scanning probe- technique (SPM) showed that all of these viruses have distinguishable and characteristic capacitances, respectively. Series of control experiments were carried out using mutant- viruses in order to validate the origin of the characteristic capacitance responses for different viruses.

A mutation on the capsid in HSV1 virus with green fluorescence- proteins (GFP) increased capacitance from $9 \times 10-6 \mathrm{~F} / \mathrm{cm} 2$ to $1 \times 10-5 \mathrm{~F} / \mathrm{cm} 2$ at the frequency of 104 Hz. HSV2 virus decreased capacitance when its envelope and glycoproteins were chemically- extracted. These control experiments indicate that dielectric properties of capsid proteins and envelope glycoproteins significantly influence overall dielectric constants of viruses. Because those capsid proteins and glycoproteins are characteristic to the virusstrain, this technique could be applied to detect and identify viruses at the single viron- level using their distinct capacitance spectra as fingerprints without labeling [13].

Eric M Kettleson, et al: "Airborne- virus capture and inactivation were studied in an electrostatic -precipitator (ESP) at applied voltages from -10 to $+10 \mathrm{kV}$ using aerosolized bacteriophages T3 and MS2. For each charging scenario, samples were collected from the effluent air stream and assayed for viable phages using plaque assays and for nucleic acids using quantitative polymerase chain -reaction (qPCR) assays. At higher applied voltages, more virus particles were captured from air with maximum- log reductions of 6.8 and 6.3 for the plaque assay and 4.2 and 3.5 for the qPCR assay at $-10 \mathrm{kV}$ for T3 and MS2, respectively.

Beyond corona inception (at applied voltages of $-10,-8,+8$, and $+10 \mathrm{kV}$ ), log reduction values obtained with the plaque assay were much higher compared to those of the qPCR assay because nonviable particles, while present in the effluent, were un accounted for in the plaque -assay. Comparisons.

Of these assays showed that in-flight inactivation (inactivation without capture) was greater for the highest applied voltages with a log inactivation of 2.6 for both phages at $-10 \mathrm{kV}$.

We have demonstrated great potential for virus -capture and inactivation via continual ion and reactive species bombardment when conditions in the ESP are enforced to generate a corona discharge" [14].

Christin Scheller, et al: "The isoelectric -point (abbreviated by $\mathrm{pI}$ or IEP) is the $\mathrm{pH}$ value at which a molecule's or biocolloid's net charge is zero. The latter includes viruses, which can form different surface charges, depending on the $\mathrm{pH}$. Information about the isoelectric- point is important because the solubility and electrical repulsion are lowest at the pI. the tendency to aggregation and precipitation is highest. In the case of viruses, the value thus provides information about the viral
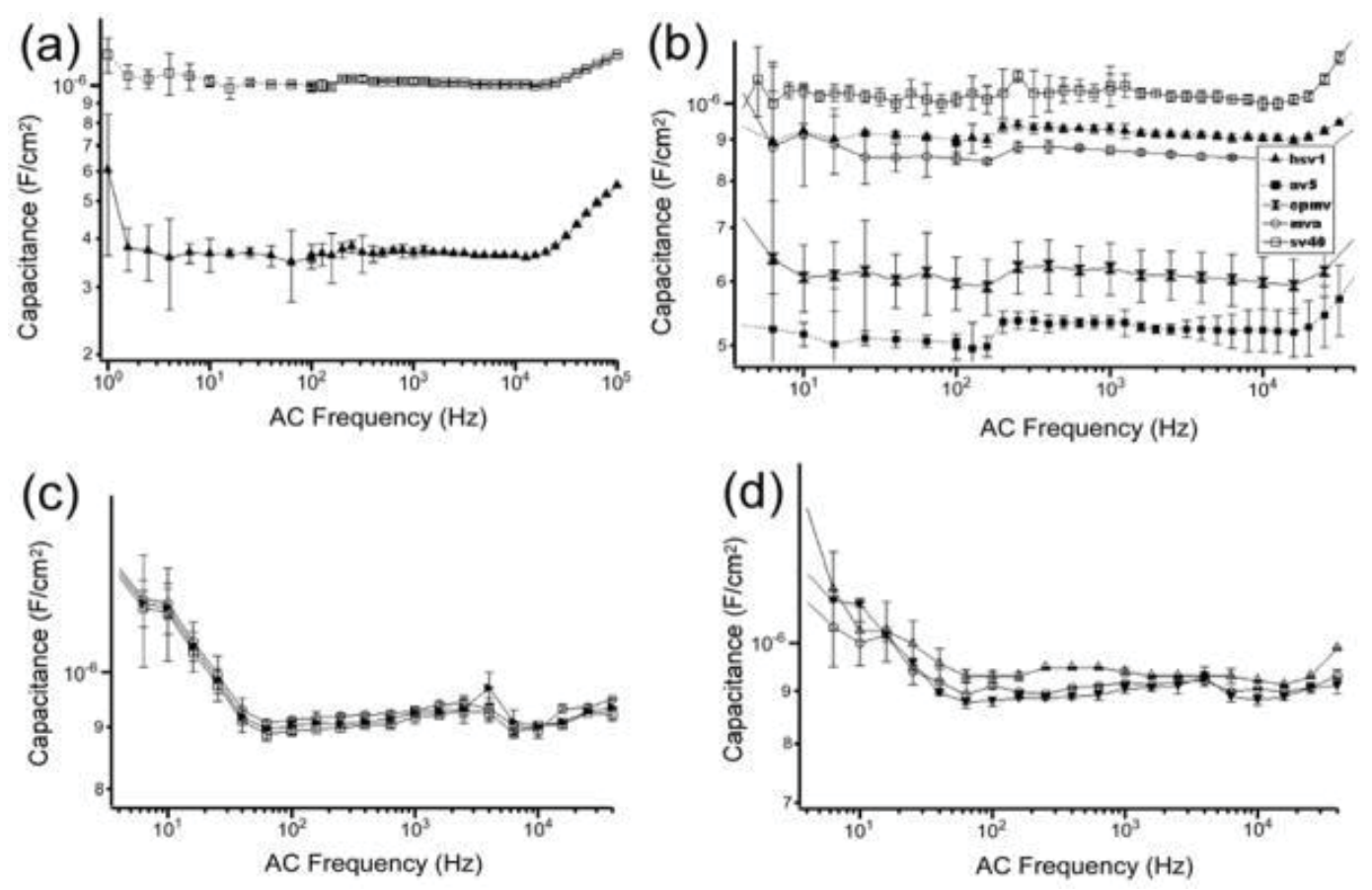

Figure 9: Capacitance -spectra of (a) polystyrene ( $\square$ ) and PMMA $(\boldsymbol{\Delta})$ nanoparticles (b) Capacitance spectra of AV5, CPMV, MVA, SV40, and HSV1 (c) HSV1 with the tip forces, $50 \mathrm{nN}(\square), 200 \mathrm{nN}(\square)$, and $500 \mathrm{nN}(\circ)$ (d) HSV1 at humidity -level $10 \%(\triangle), 50 \%(\circ)$, and $70 \%(\boldsymbol{\nabla})$ ). shows capacitances of five types of viruses; adenovirus type 5 (AV 5), herpes simplex- virus type 1 (HSV1), simian virus 40 (SV40), vaccinia (MVA), and cowpea mosaic virus -(CPMV). Their impedance signals were averaged $1-10$ seconds for each AC frequency at $10 \mathrm{mV}$ of $\mathrm{AC}$ bias. For each type of virus, fifty different particles were measured and collected capacitance- spectra were averaged with error bars and normalized by the size of each virus, as shown in Figure (b). In this figure, SV40 showed the highest capacitance while AV5 had the lowest. 
surface charge in a specific environment. Michen and Graule authored a review on isoelectric- points of viruses in which they evaluated $137 \mathrm{pI}$ measurements of 104 viruses. Their evaluation shows that the isoelectric- points (IEP) of viruses range from 1.9 to 8.4, but can mostly be found between 3.5 and 7 , which means that viruses with pI values in the strongly basic range have not yet been described.

They offer various attempts to explain the variations found in single-virus species data. Key propositions are that viruses can have more than one $\mathrm{pI}$ and that the pI value depends on the electrolyte- conditions to which they are exposed. Dependencies of charge states, and thus, pI values, on the presence of metal ions (for example) have been frequently observed. At present, no experimental data are available for the $\mathrm{pI}$ value of SARS-CoV-2 or its structural, nonstructural, or accessory- proteins. An estimation is possible through predictive calculations with, in example, the ProtParam tool by ExPASy. The calculation results for the physico-chemical properties and the molecular weight of the individual proteins are based on the corresponding amino acid sequences from UniProt" [15].

"The (pKa)s of the protein on the surface: At the isoelectric -point of a protein, there is an equivalent distribution of negative and positive- charges, leading to a neutral response to a potential difference. If the $\mathrm{pH}$ be lowered from the isoelectric -point, it is found that, a bias is created towards the centre of positive charge of the protein. The pKa's could be derived from the $\mathrm{pH}$ values of the titration curve for the S(spike)-protein of the SARS-CoV-2 using the HendersonHasselbalch equation (from titration analysis):

$\mathrm{pH}=\mathrm{pKa}+\log ([$ conjugate base $] /[$ weak acid] $])$ To establish this concept of the influence of surrounding $\mathrm{pH}$ on the surfacecharge of a virus, similar experiments had already been carried out also over the other virus strains. It should be considered here that the S-protein in the spikes of the coronavirus is essentially a complex folded structure of a chain formed from the the $\mathrm{S}$ gene that comprises an ORF encoding a protein of 1353 amino acid- residues, with a predicted molecular weight of 149,918 (S Mounir 1, P Labonté, P J Talbot).

It tends to have different charge accumulations and configurations in different $\mathrm{pH}$ levels of the surroundings, unlike simple inorganic- particles of aerosols. A three layer mask can be produced to prevent the immediate infections of DNA, RNA viruses including SARS-COV-2 and others because the electric- charge accumulation of the RNA viruses is much less than the electrostatic charges accumulated in the layers of the mask within few minutes. In the inner surface, a cotton type non- conducting material can be used which will work basically as a nonconductor so that the electrostatic -charges produced inside the two layers does not drain out through surface of the (human)body" [16].

Marie Hagbom, et al: "Generation of negative- ions has previously been shown to reduce transmission of Newcastle disease virus 22,23 and several kind of bacteria24,25 in animal experimental set-ups.

By the use of a modified ionizer- device we describe effective prevention of airborne transmitted influenza- A (strain Panama 99) virus infection between animals and inactivation of virus (> 97\%). Active ionizer prevented 100\% (4/4) of guinea -pigs from infection. the device effectively captured airborne transmitted calicivirus, rotavirus and influenza- virus, with recovery rates up to $21 \%$ after $40 \mathrm{~min}$ in a $19 \mathrm{~m} 3 \mathrm{room}$. The ionizer generates negative- ions, rendering airborne- particles/aerosol droplets negatively charged and electrostatically attracts them to a positively charged collector plate. Trapped viruses are then identified by reverse transcription quantitative real-time PCR. The device enables unique possibilities for rapid and simple removal of virus from air and offers possibilities to simultaneously identify and prevent airborne- transmission of viruses. Inactivation of viruses by electrostatic attraction has only been briefly investigated. In the present study, rotavirus and CaCV lost significant ( $>97 \%$ ) infectivity (ratio; CaCV from 3.0 $\times 10-2$ to $<7.8 \times 10-4$ and rotavirus from $4.9 \times 10-1$ to $<7.6 \times 10-3$ ) in ionized- air as determined by a ratio of infectivity versus gene -copies. The ionizer creates electrons, which will render surface molecules of particles in air negatively -charged thus attracting them to the positively charged collector plate. This device generates approximately 35000 billion electrons per second with a steady-state ozone concentration below the detection limit $(0,002 \mathrm{ppm})$ as tested by VTT Technical Research Center of Finland, Tampere" [17].

An isoelectric point is the point at which a particular molecule carries no net electric -charge. Thus at isoelectricpoint the total positive and negative charges are same and the molecule is overall neutral. If a protein has more number of acidic amino -acids, the isoelectric -point will be at low $\mathrm{pH}$ while a protein with more number of basic amino acids will have isoelectric point at high $\mathrm{pH}$.

Lin L, et al: "Electrostatic -features, including electrostatic potential, electric -field lines, and electrostatic forces of SARS$\mathrm{CoV}$ and SARS-CoV-2 were calculated and compared in detail.

mowhen

The charge on an amino acid depends on the pH.

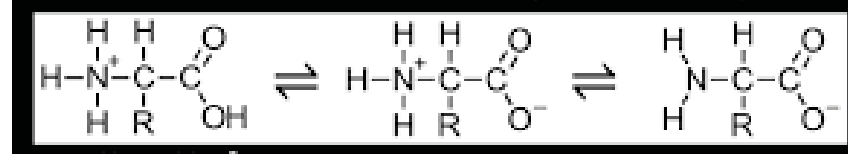

$\mathrm{pH}<\mathrm{pH}$ of

Isoelectric point

pH > pH of

isoelectric point

cation

isoelectric point

The isoelectric point is the $\mathrm{pH}$ at which an amino

acid is electrically neutral. 
The results demonstrate that SARS-CoV and SARS-CoV-2 S proteins are both attractive to ACE2 by electrostatic forces even at different distances. the residues contributing to the electrostatic- features are quite different due to the mutations between SARS-CoV S protein and SARS-CoV-2 S protein. Such differences are analyzed comprehensively" [19].

Red represents residues which are mutated to be more negative; Blue represents residues which are mutated to be more positive; yellow represents residues which are mutated from polar to hydrophobic; cyan represents residues which are mutated from hydrophobic to polar. (B) Structure of a single $\mathrm{S}$ protein monomer. The RBD shown in red circle is flipping out to reach ACE2. The green circle- region highlights the hinge between RBD and the rest of S protein.

Zhi-Yong Wang, et al: "Bronchiolitis, a common lower respiratory- tract infection in infants, is the primary reason of hospitalization of infants in developed and developing -countries (1). This disease is characterized by wheezing, cough and tachypnea. Cases mostly present among the infants aged 1-6 months. It usually occurs in early spring and winter seasons.

"It is estimated that 1 in 5 infants each year suffers respiratory- infection caused by respiratory syncytial virus (RSV) (3). The mortality rate is $0.5 \%-1.5 \%$ among hospitalized infants, but increases to $3-4 \%$ for infants with potential pulmonary or cardiac- diseases.

A meta-analysis was performed to analyze the efficacy of 3\% hypertonic -saline (HS) in bronchiolitis. Pubmed and MEDLINE databases were searched for relevant articles. A total of 2 authors selected the articles according to the inclusion criteria and then data were carefully extracted. Weighted mean difference (WMD) with 95\% confidence interval $(95 \% \mathrm{CI})$ values were used to pool continuous data, including length of stay and clinical severity- score (CSS). Relative- risk (RR) with 95\% CI was calculated to determine the association between 3\% HS and re-admission. The pooled data revealed that infants treated with 3\% HS exhibited shorter durations of hospitalization compared with those

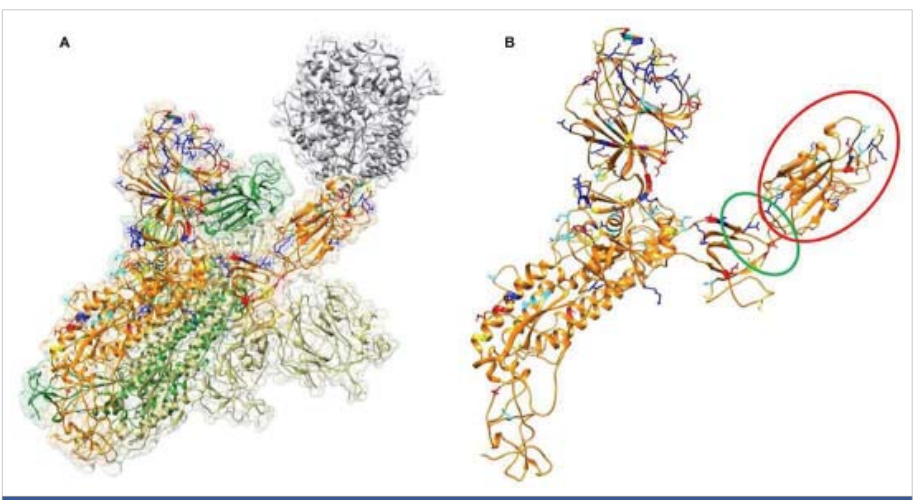

Figure 11: Structure of SARS-CoV-2 S proteins and ACE2 binding- domain. (A) The structure of $S$ protein trimer binding with ACE2 binding- domain. ACE2 is shown in gray color. treated with normal- saline (NS; WMD $=-0.43$; 95\% CI = $-0.70,-0.15)$. Subgroup -analysis examining the combination of HS or NS with additional medication demonstrated that $3 \%$ HS with epinephrine significantly decreased the length of hospital stay, with a WMD $=-0.62(95 \%$ CI $=-0.90,-0.33)$. The results indicated a lower CSS score in the 3\% HS group compared with the NS group (SMD $=-0.80 ; 95 \% \mathrm{CI}=-1.06$, $-0.54)$. The pooled outcome indicated a beneficial effect of $3 \%$ HS on decreasing re-admission rates compared with NS (RR = 0.93 ; $95 \% \mathrm{CI}=0.70,1.23$ ). No potential publication- bias was observed (Begg's, $p=0.133$; Egger's, $p=0.576$ ). In conclusion, $3 \%$ HS was demonstrated to be a more successful therapy compared with NS for infants with bronchiolitis" [20].

C M Schaldach, et al: "The influence of ionic strength on the electrostatic -interaction of viruses with environmentally relevant surfaces was determined for three viruses, MS2, Q beta, and Norwalk. The virus is modeled as a particle comprised of ionizable amino acid- residues in a shell surrounding a spherical RNA core of negative charge, these charges being compensated for by a Coulomb screening due to intercalated ions. A second model of the virus involving surface charges only is included for comparison. Surface potential calculations for each of the viruses show excellent agreement with electrophoretic- mobility and zeta potential measurements as a function of $\mathrm{pH}$. The environmental- surface is modeled as a homogeneous plane held at constant potential with and without a finite region (patch) of opposite potential. The results indicate that the electrostatic- interaction between the virus and the oppositely- charged patch is significantly influenced by the conditions of ionic strength, $\mathrm{pH}$ and size of the patch. Specifically, at pH 7, the Norwalk -virus interacts more strongly with the patch than MS2 (approximately 51 vs. approximately $9 \mathrm{kT}$ ) but at $\mathrm{pH} 5$, the Norwalk-surface interaction is negligible while that of MS2 is approximately $5.9 \mathrm{kT}$. The resulting ramifications for the use of MS2 as a surrogate for Norwalk are discussed" [21].

Preprint: Rafael RG. Machado et al: "We performed experiments to test if hypertonic saline solution is able to inhibit virus- replication in vitro. Our data shows that $260 \mathrm{mM}$ $\mathrm{NaCl}(1.5 \%)$ inhibits $100 \%$ SARS-CoV-2 replication in Vero cells. our results suggest that the virus replication inhibition is due to an intracellular -mechanism and not due to the dissociation between spike SARS-CoV-2 protein and its humanreceptor angiotensin-converting enzyme 2 interaction. $\mathrm{NaCl}$ depolarizes the plasma membrane supposedly associated with the inhibition of the SARS-CoV-2 life cycle.

This observation could lead to simple, safe and low cost interventions at various stages of COVID-19 treatment, improving the prognosis of infected- patients, thereby mitigating the social and economic costs of the pandemic" [22]. 
Srinivasan Damodaran: Salts and protein Denaturation.

"Salts affect protein stability in 2different ways. At lowconcentrations, ions interact with proteins via nonspecific electrostatic- interactions. This electrostatic neutralization of protein charges usually stabilizes protein structure. Complete charge neutralization by ions occurs at or below $0.2 \mathrm{M}$ ionic strength and it is independent of the nature of the salt. At higher- concentrations ( $>1 \mathrm{M})$, salts have ion specific effects that influence the structural -stability of proteins."

\section{Conclusion}

Related the literature reported it is interesting to submit to the researcher that it is possible acts on the Electrical distribution of a respiratory virus surface.

This modulation can help in various stage of the pathogenetic process since form the binding with the ACE receptor, to the cellular intake.

Is I reported the effect of hypertonic saline solution in aerosols as medium that can modify this equilibrium.

In some example of pediatric disease reducing the days of pathologic condition.

This is a simply example able to show as modifying the microenvironment in the interface whit an aerosolized ionic substantia this can create an unfavorable situation for virus cellular intake.

Hypertonic saline solution aerosol show more efficacy the normal saline and this is the proof that the;

Increase Ionic force is crucial for the effect.

Adding the salts increase ionic forces.

Can we use other substantie for the same scope and more effective acting on micro environmental factors like ionic forces, osmotic level, $\mathrm{PH}$ and other affecting the global protein structure?

Ethical consideration: Under all international rules.

Clarification this work is produced whit out any diagnostic or therapeutic intent only to submit to researcher hypothesis of work.

\section{Ionic Strength(I)}

Ionic Strength (I) is a measure of the electrical intensity of a solution containing ions.

- Mathematically.

$$
I=\frac{1}{2} \sum_{i=1}^{i=n} c_{i} z_{i}^{2}
$$

\section{References}

1. Rakshita K, Chatterjeea S, Bandyopadhyaya D, Sarkarb S. Preprint an effective approach to reduce the penetration potential of sarscov-2 and other viruses by spike protein: surface particle.

2. Schoeman D, Fielding BC. Coronavirus envelope protein: current knowledge. Virol J. 2019; 16: 69.

PubMed: https://pubmed.ncbi.nlm.nih.gov/31133031/

3. Northwestern University Amanda Morris Research exposes new vulnerability for SARS-CoV-2. Electrostatic interactions enhance the spike protein's bond to host cells. 2020.

4. Leung WWF, Sun Q. Electrostatic charged nanofiber filter for filtering airborne novel coronavirus (COVID-19) and nano-aerosols. Sep Purif Technol. 2020; 250: 116886.

PubMed: https://pubmed.ncbi.nlm.nih.gov/32322159/

5. Sholanov K. Electrophysical processes effecting the covid-19 virus before it enters. Organism.

6. Li W. Structurally Observed Electrostatic Features of the COVID-19 Coronavirus-Related Experimental. Structures inside Protein Data Bank: A Brief Update.

7. Luisetto M. Chemico- physicals properties of coronavirus affecting. Int Invent Sci J. 2021; 5:

8. Baron J, El-Chaar G. Hypertonic Saline for the Treatment of Bronchiolitis in Infants and Young Children: A Critical Review of the Literature. J Pediatr Pharmacol Ther. 2016; 21: 7-26.

PubMed: https://pubmed.ncbi.nlm.nih.gov/26997926/

9. Biktasheva IV. Role of a habitat's air humidity in Covid-19 mortality. Sci Total Environ. 2020; 736: 138763.

PubMed: https://pubmed.ncbi.nlm.nih.gov/32492610/

10. Jing JLJ, Yi TP, Bose RJC, McCarthy JR, Tharmalingam N, et al. Hand Sanitizers: A Review on Formulation Aspects, Adverse Effects, and Regulations. Int J Environ Res Public Health. 2020; 17: 3326. PubMed: https://pubmed.ncbi.nlm.nih.gov/32403261/

11. Pawłowski PH. Charged amino acids may promote coronavirus SARSCoV-2 fusion with the host cell. AIMS Biophysics. 2021; 8: 111-120. PubMed: http://www.aimspress.com/journal/biophysics

12. Al Ahmad M, Mustafa F, Ali LM, Rizvi TA. Virus detection and quantification using electrical parameters. Sci Rep. 2014; 4: 6831. PubMed: https://pubmed.ncbi.nlm.nih.gov/25355078/

13. MacCuspie RI, Nuraje N, Lee SY, Runge A, Matsui H. Comparison of Electrical Properties of Viruses Studied by AC Capacitance Scanning Probe Microscopy. J Am Chem Soc. 2008; 130: 887-891. PubMed: https://pubmed.ncbi.nlm.nih.gov/18092777/

14. Kettleson EM, Ramaswami B, Hogan CJ, Jr., Lee MH, Statyukha GA, et al. Airborne Virus Capture and Inactivation by an Electrostatic Particle Collector. Environ Sci Technol. 2009; 43: 5940-5946. PubMed: https://pubmed.ncbi.nlm.nih.gov/19731701/

15. Scheller C, Krebs F, Minkner R, Astne I, Gil-Moles M, et al. Physicochemical properties of SARS-CoV-2 for drug targeting, virus inactivation and attenuation, vaccine formulation and quality control. 2020.

16. Hagbom M, Nordgren J, Nybom R, Hedlund KO, Wigzell $H$, et al. lonizing air affects influenza virus infectivity and prevents airbornetransmission. Sci Rep. 2015; 5: 11431.

17. Xie Y, Karki CB, Du D, Li H, Wang J, et al. Spike Proteins of SARSCoV and SARS-CoV-2 Utilize Different Mechanisms to Bind with Human ACE2. Front Mol Biosci. 2020; 7: 591873.

PubMed: https://www.ncbi.nlm.nih.gov/pmc/articles/PMC7755986/

18. Wang ZY, Li XD, Sun AL, Fu XQ. Efficacy of $3 \%$ hypertonic saline in bronchiolitis: A meta-analysis. Exp Ther Med. 2019; 18: 1338-1344. PubMed: https://pubmed.ncbi.nlm.nih.gov/31384334/ 
19. Schaldach CM, Bourcier WL, Shaw HF, Viani BE, Wilson WD. The influence of ionic strength on the interaction of viruses with charged surfaces under environmental conditions. J Colloid Interface Sci. 2006; 294: 1-10.

PubMed: https://pubmed.ncbi.nIm.nih.gov/16083898/

20. Rafael RG. Machado F, Glaser T, Araujo DB, Petiz LL, et al. Hypertonic saline solution inhibits SARS-CoV-2 in vitro assay.
21. Rakshit K. An Effective Approach to Reduce the Penetration Potential of SARS-CoV-2 and other Viruses by Spike Protein. AIJR Preprints. 2020.

22. Morton SP, Phillips JL. Computational Electrostatics Predict Variations in SARS-CoV-2 Spike and Human ACE2 Interactions. BioRxiv. 2020. 